\title{
Carbon Vapor and Carbon Plasma Infrared Emission
}

\section{Laszlo Nemes*}

Research Centre for Natural Sciences, Hungarian Academy of Sciences, Institute of Materials and Environmental Chemistry, Magyar Tudósok körútja 2, Budapest 1519, Hungary

\begin{abstract}
A review is given about infrared emission spectroscopy of hot carbon vapors and plasmas obtained using Fourier transform infrared emission and laser induced breakdown spectroscopy (LIBS) in the mid-infrared range. Laboratory FTIR emission spectra contain vibrational bands from fullerenes $C_{60}, C_{70}$ whereas laboratory mid-infrared LIBS spectra show bands that belong to mostly unidentified carbon molecules and clusters. Both kinds of spectra are compared to spectral results from infrared astronomy. The spectra are discussed with a view for possible applications in carbon nanostructure research and in infrared astronomy. Extensions for laser induced breakdown (plasma) spectroscopy in the infrared range are suggested.
\end{abstract}

Keywords: Carbon plasma; Carbon vapor; Infrared emission; FTIR; LIBS; Laser-induced

\section{Introduction}

Even before the discovery of fullerenes [1], and their macroscopic production [2], interest arose in finding carbon molecules (e.g. linear carbon chains) in the interstellar medium, in carbon rich stars and in other cosmic sources. Carbon is an abundant chemical element in the Cosmos [3] and is of central importance on Earth from several essential points of view. Thus the discovery of the interesting new allotropes of carbon $[1,4]$ initiated an exponentially growing scientific literature that by now covers a great diversity of chemical and physical disciplines. One interesting subfield is the search for carbon containing molecules in carbon-rich stars [5]. With the developments of infrared facilities on boards of space telescopes and available on ground based infrared observatories detection and identification of carbon molecules became possible.

Identification of large and small carbon molecules is generally straightforward in infrared spectra, especially in the mid-infrared range, as they contain molecular 'fingerprints' used in physical organic and analytical chemistry in molecular structure research. It is obviously important to study the infrared emission spectra of carbon molecules in the laboratory in order to obtain sample spectra to compare to observations, e.g. by astronomical means. Such infrared spectra may be obtained by several techniques. One important method to augment emission studies is to use cryogenic temperature rare gas matrix isolation that provides absorption spectra of isolated solid phase molecules, On the emission side one can generate carbon vapors and study their infrared emission from gas-phase molecules. Another new approach is the application of laser-induced plasma breakdown emission spectroscopy (LIBS) to study carbon plasmas. Plasma spectra are related to the spectra of molecules isolated in the gaseous phase and differ in several aspects from cryogenic matrix spectra, for example in relative band intensities.

The technique of LIBS is widespread in atomic and diatomic spectroscopy as such electronic transitions are mostly observed in the visible and ultraviolet spectral ranges. However conventional LIB spectra of carbon plasmas in these spectral domains do not yield molecular information for $\mathrm{C}_{3}$ and larger carbon molecules. (Although they are usually rich in $\mathrm{C}_{2}$ features, that in turn cannot be observed in vibrational spectra). The electronic transitions of larger carbon molecules are extremely difficult to observe under the usual LIBS conditions.
In recent times a new method has been elaborated for the observation of mid-infrared emission from laser generated plasmas [6-9] that was extended to carbon plasmas [10]. Although application to carbon is still in early developmental stage it has already provided preliminary results that will be discussed in this paper with a view of their applicability for materials science research as well as for infrared astronomy.

\section{Experimental Method}

There were several spectroscopic methods reviewed in this paper. Laboratory methods were Fourier-transform infrared emission spectroscopy and laser-induced breakdown spectroscopy in the mid-infrared range. FTIR spectra were taken on the National Solar Observatory Fourier-transform spectrometer at Kitt Peak, Arizona, USA, while mid-infrared LIB spectra at Hampton University, Department of Physics, Hampton, Virginia, USA. Further experimental data are given in sections 1, 2 and 3 below. Infrared astronomical spectra referred to in the text were taken by the Infrared Spectrograph on the NASA Spitzer Space Telescope.

\section{Infrared emission from hot carbon vapors}

Gas-phase infrared emission spectra of fullerenes were reported $[11,12]$ and the temperature-dependence of the vibrational bands of $\mathrm{C}_{60}$ and $\mathrm{C}_{70}$ were determined [12]. Solid fullerenes were evaporated in a high temperature furnace and the infrared emission was recorded by a Fourier-transform spectrometer. Together with previous studies of temperature-dependence in solid state spectra of fullerenes [13] and theoretical calculations for infrared and Raman modes [14] enough experimental data have been accumulated for vibrational identification of $\mathrm{C}_{60}$ and $\mathrm{C}_{70}$ in various media and physical states. The $\mathrm{C}_{60}$ molecule has four infrared active $\left(\mathrm{t}_{1 \mathrm{u}}\right)$ bands at $1404\left(v_{25}\right), 1167\left(v_{26}\right), 570\left(v_{27}\right)$ and

*Corresponding author: Laszlo Nemes, Research Centre for Natural Sciences, Hungarian Academy of Sciences, Institute of Materials and Environmental Chemistry, Magyar Tudósok körútja 2, Budapest 1519, Hungary, Tel: +36 1382 6200; E-mail: nemes.laszlo@ttk.mta.hu

Received December 04, 2017; Accepted December 26, 2017; Published December 29, 2017

Citation: Nemes L (2017) Carbon Vapor and Carbon Plasma Infrared Emission. J Astrophys Aerospace Technol 5: 154. doi:10.4172/2329-6542.1000154

Copyright: @ 2017 Nemes L. This is an open-access article distributed under the terms of the Creative Commons Attribution License, which permits unrestricted use, distribution, and reproduction in any medium, provided the original author and source are credited. 
$528\left(v_{28}\right) \mathrm{cm}^{-1}(7.1,8.6,17.5$ and $19 \mu \mathrm{m}$, resp. $)$ at about $680^{\circ} \mathrm{C}$ in the gas phase, whereas $\mathrm{C}_{70}$ has eight infrared active $\left(\mathrm{e}_{1}\right.$ ' or $\mathrm{a}_{2}$ ") bands at 1414 $\left(v_{24}\right), 1125\left(v_{30}\right), 1077\left(v_{79}\right), 793\left(v_{33}\right), 639\left(v_{36}\right), 575\left(v_{37}\right), 558\left(v_{35}\right)$, and $529\left(v_{82}\right) \mathrm{cm}^{-1}(7.0,8.9,9.3,12.6,15.6,17.4,17.9$ and $18.9 \mu \mathrm{m}$, resp.) around $700^{\circ} \mathrm{C}$.

Using the temperature dependence of the vibrational bands of $\mathrm{C}_{60}$ a rough extrapolation to absolute zero temperature was done [12] and the results agreed among these extrapolated values and those observed in argon matrix isolation spectra [15]. Thus data derived from hot vapors may also be useful for the observation of very cold gas-phase fullerenes in astronomical sources. In fact these extrapolated values and matrix isolation data were used for the identification of fullerenes $\mathrm{C}_{60}$ and $\mathrm{C}_{70}$ in planetary nebulae $[16,17]$ using the Infrared Spectrograph on board of the Spitzer Space Telescope [18].

\section{Mid-infrared emission from laser-generated plasmas}

Previous studies of time-resolved infrared emission from laserinduced breakdown spectroscopy of inorganic materials [6-9] led to the development of the necessary instrumentation and methodology. This is a significantly new extension of the traditional LIB methods. These studies were and are carried out for remote sensing of chemical, biological and explosive materials, such as various alkali nitrates, ammonium perchlorate and gunpowder [19], and various simple pharmaceuticals such as Tylenol and Aspirin, and compounds like dimethyl phosphate and methyl salycilate [20].

To detect infrared emission in the mid-infrared spectral range the latest instrumentation applied a flash-lamp pumped Q-switched Nd: YAG laser at its fundamental wavelength $1064 \mathrm{~nm}$, and for spectral detection a cooled MCT linear detector array using time-resolved electronics allowing time-gating $[19,20]$. Linear detector arrays make it possible to run spectra in a few seconds. Previous experiments applied a single element MCT detector and a scanning grating spectrometer, thus were much slower.

Developments of mid-infrared LIBS created the possibility for observing time-resolved infrared emission from laser-generated carbon plasmas. These experiments are somewhat different from analogous remote-sensing studies on energetic materials and pharmaceuticals, as while the ablation laser evaporates or ablates existing molecules from inorganic and organic samples, in the carbon plasma experiments the only readily available molecular entities could only be laser-ablated graphite lattice fragments and macroscopic graphite particles. However many of the signatures observed in carbon plasma infrared emission likely belong to vibrational bands of laser-generated carbon molecules.

\section{Study of carbon plasmas by mid-infrared LIBS}

In a recent paper the results of the application of the LIBS technique to study time-resolved infrared emission from carbon plasmas are described in detail [10]. Here the observation of such spectra is summarized and some possible improvements and developments are outlined. The following is a short summary of experimental details, full experimental description is given [10].

The infrared LIB spectra obtained for carbon were obtained at the Department of Physics, Hampton University, Hampton, Virginia, USA with contributions from Battelle Eastern Sciences and Technology Center, Aberdeen, Maryland, USA. The spectra were run between 7.5 and $11.5 \mu \mathrm{m}$, and in one case between 4.5 and $11.5 \mu \mathrm{m}$. A grating spectrometer of $150 \mathrm{~cm}$ focal length was used, providing about $80 \mathrm{~nm}$ spectral resolution. Detection was made by a cooled $\mathrm{HgCdTe}$ (MCT) detector at $77 \mathrm{~K}$. The spectral sensitivity of the detector was at maximum at 10 micron, near $40 \%$ at 4 micron, and near $70 \%$ at 12 micron. Infrared radiation from target plasma was focused by $\mathrm{ZnSe}$ optics onto the $2 \mathrm{~mm}$ wide entrance slit of the spectrometer. Time resolution made it possible to use spectral delays 10-20 microsecond relative to the laser pulse. Scattered laser light and higher-order spectra were cut using lowpass filters at 4 and 7.4 micron, total spectral taking time was near 1 hour. The spectrometer was encased in a plastic box that was flushed by ultra-pure grade $\mathrm{N}_{2}$, Ar or Heat atmospheric pressure.

The infrared spectra contained broad emission bands. All spectra were characterized by strong continuous backgrounds. Spectra recorded in the $8-11.5 \mu \mathrm{m}$ interval were weaker than those in the 4.5 $-8 \mu \mathrm{m}$ region. The broadness of the emission bands is due to a lesser extent to the limited spectral resolution (about $80 \mathrm{~nm}$ ) but mainly to the high target and plasma temperature, the fundamental source for their width is however their molecular origin. At plasma temperatures vibrational and rotational excitations are extensive leading to broad spectral envelopes. Atomic lines would have been much narrower. In addition, in the infrared region studied no neutral and/or ionic atomic lines are expected to appear with significant intensity [21].

The observed infrared emission did not show the room temperature blackbody emission expected from the spectrometer body itself, and significant bands were only observed in atmospheric pressure rare gases. In ref. [10] it was found that emission spectra were somewhat different in argon and in helium. Figure 1 below shows a comparison in the range $7.4-11.5 \mu \mathrm{m}$. The spectra shown were bckground corrected and denoised using methods described [10].

This difference suggests molecule and cluster formation in the plasma. However not all spectral features may be due to molecule formation in the plasma, as the laser ablates graphene fragments from the graphite target. In addition, macroscopic graphite particles may be ablated or could form within the plasma. Graphene fragments are dehydrogenated PAH (dPAH) molecules, i.e., condensed aromatic carbon rings. Infrared bands of $\mathrm{dPAH}$ molecules computed using high level density functional methods were reported in the literature [22]. The strongest infrared bands computed for $\mathrm{DPAH}$ molecules lie between 5 and $12 \mu \mathrm{m}$ and between 17 and $28 \mu \mathrm{m}$. dPAH molecules are essentially nano-graphene particles, and in a theoretical vibrational study they were found to possess complicated vibrational phonon modes in the mid-infrared region, as calculated by atomistic methods using a modified Brenner potential [23]. For example, nanographene

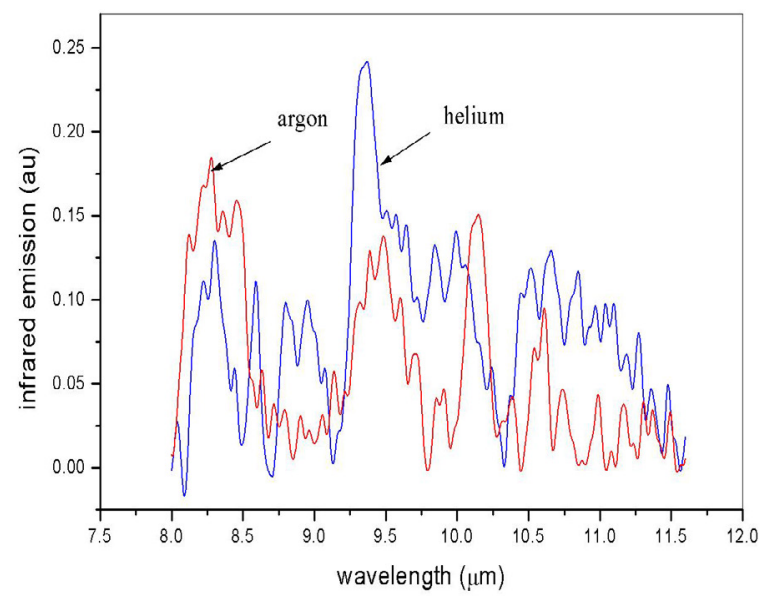

Figure 1: Comparison of carbon plasma spectra in helium and argon background gases. 


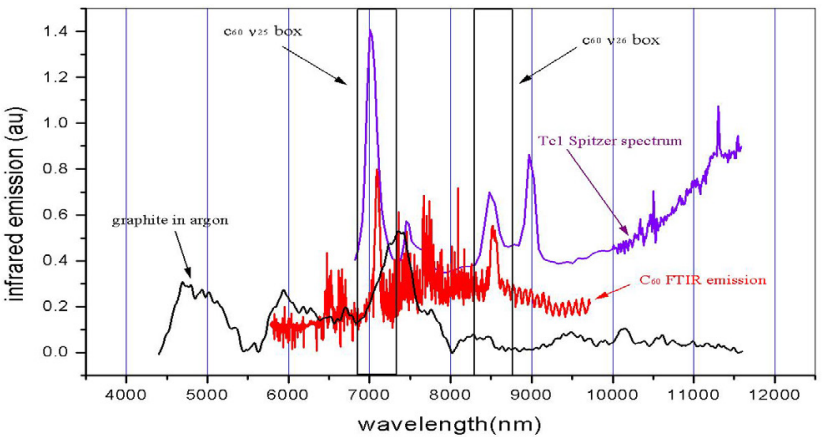

Figure 2: Spectral comparison among laboratory infrared emission, laboratory mid-infrared LIB and Spitzer telescope MIR spectra.

molecules with 53 carbon atoms have high phonon densities between 200 and $2000 \mathrm{~cm}^{-1}(50-5 \mu \mathrm{m})$. Perfectly regular graphene with 1600 atoms has a well isolated strong phonon density peak at around $5.9 \mu \mathrm{m}$ [24].

In addition, the strong band observed between 4.4 and $5.4 \mu \mathrm{m}$ does likely contain vibrational features of $\mathrm{C}_{3}, \mathrm{C}_{5}, \mathrm{C}_{6}, \mathrm{C}_{7}$ and $\mathrm{C}_{9}$ linear carbon chain molecules, as expected from their matrix isolation infrared spectra [25].

An interesting possibility is that graphite target laser ablation plasmas contain the fullerenes $\mathrm{C}_{60}$ and $\mathrm{C}_{70}$. The availability of laboratory hot carbon vapor infrared spectra and infrared astronomical observations provide comparisons. Such a comparison is shown in Figure 2.

In the above Figure black denotes mid-infrared LIB spectra [10], magenta denotes the spectrum from the Spitzer telescope [18] (the author acknowledges Professor Jan Cami for sending the digital form of the spectrum), while red denotes infrared spectra from hot $\mathrm{C}_{60}$ vapor [12]. As shown the $v_{25}$ and $v_{26}$ infrared modes of $\mathrm{C}_{60}$ at around 7 and 8.5 micron overlap between the laboratory $\mathrm{C}_{60}$ vapor and the Spitzer infrared spectra, but the mid-infrared LIB spectra do not have prominent bands at these wavelengths (see the boxes in the figure bracketing the two fundamental modes of $\mathrm{C}_{60}$ ). The infrared modes of $\mathrm{C}_{70}$ also fall in the wavelength range of Figure 2. The mid-infrared LIB spectra however contain strong emission bands at around 4.7, 6 and 7.4 microns. None of these correspond to $\mathrm{C}_{60}$ or $\mathrm{C}_{70}$ infrared bands, or those of linear carbon clusters. The 7.4-micron band might contain the infrared signatures of dPAH molecules (nanographenes).

\section{Discussion and Propositions}

Infrared emission spectra observed by the mid-infrared LIBS (MIRLIBS) method are at present difficult to assign with any certainty to known carbon molecules. As mentioned before, there are only two groups of molecules (linear carbon chains and dehydrogenated PAHs) for which previously obtained experimental data and highlevel theoretical calculations provide a tentative basis for identification. Fullerenes $\mathrm{C}_{60}$ and $\mathrm{C}_{70}$ could not yet been positively identified in MIRLIB spectra and there are strong emission bands for which at present no molecular assignments could be given.

The present results from mid-infrared LIB spectra of carbon plasmas [10] are thus only preliminary as the experiments were performed using an equipment designed for observing inorganic and organic molecular matter for remote-sensing analytical applications under atmospheric conditions.
In order to exploit this new analytical technique, the method should be extended and refined. Relative to the published spectra [10] obtained with a single element MCT ( $\mathrm{HgCdTe})$ detector/ scanning spectrometer requiring up to 1 hour of recording 10 spectra, the newly developed linear array MCT detector/grating based monochromator should provide fast acquisition of the infrared spectra (only 5 secs is needed to average 4 single-shot plasma spectra [19]). Thus, with the linear array detector many spectra can be averaged resulting in significantly improved signal to noise ratio.

Another aspect is the time-resolved ability of the experimental arrangement. While in the previous experiments [10] time-delays up to 20 microseconds after the laser-pulse were used, in the experiments with the linear array time resolution was extended to $1 \mathrm{~ms}$ delay. This is important e.g. for observing fullerenes as their formation in the plasma requires time scales longer than 10-20 microseconds. In addition, one would need warm background gases for cluster formation compared to the room temperature surrounding gas in previous experiments [10].

Increase of spectral resolution greater than $80 \mathrm{~nm}$ in further experiments would bring obvious advantages. An important extension of MIRLIB spectra of carbon and other substances would be the variation of environmental gas pressure. For remote-sensing applications that often are carried out in ambient air, the necessity of using atmospheric (about $100 \mathrm{kPa}$ ) pressure is obvious, however to study the formation of atomic clusters much lower pressures are usually needed (maybe down to a few tens of Pascal).

MIRLIB spectra are dominated by a strong thermal (blackbody) background. Using ablation lasers with sub-nm pulse lengths (femtoseconds?) that have much less thermal contribution than nanosecond lasers, this thermal background could be reduced. Such fast lasers may also make it possible to separate the infrared emission from surface molecules from thermal emission from excitation of laser generated clusters.

A significant advance of MIRLIB spectroscopy could result from simultaneous use of the conventional LIBS technique (in the visible and ultraviolet region) and the MIRLIBS method. These two methods are quasi mutually exclusive. As in the infrared range atomic lines of various ionization stages are not observed, whereas in conventional LIBS atomic lines are easily used to obtain electron temperature and electron volume density values (based on relative intensities and line widths of atomic lines), quantitative characterization of the plasmas in combined LIBS spectral ranges would be possible. The present state of developments infrared LIB spectroscopy and relevant technical aspects are reviewed in a recent paper [26].

With increased spectral resolution, better signal to noise ratios, non-thermal lasers and available plasma electron parameters it might be possible to study vibrational excitation conditions, further molecular identifications and maybe derive molecular formation mechanisms (thus plasma chemistry) as well.

Developments in the experimental techniques could provide important help for understanding carbon cluster formation which is a central theme in producing various carbon nanostructures. In addition the MIRLIBS technique could be extended to other cluster forming chemical elements, such as metals, alkali metals, semi-metals and some non-metallic elements (such as sulphur), thus a large part of the periodic table. As these atomic clusters usually emit in the far infrared range MIRLIBS wavelengths should be extended. This depends on the evolution of fast far-infared detectors. Such studies may also lead to practical results for the nanomaterials field. 


\section{Conclusion}

Infrared astronomy may also benefit from carbon MIRLIB spectroscopy in identifying the molecular origin and physical state of cosmic infrared emitting sources containing carbon, along the line of the Spitzer spectra of fullerenes [16,17]. In particular with the midinfrared observational possibilities of the coming James Webb Space Telescope between 5 and 28 microns [27] one may expect extensions of the Spitzer telescope's spectroscopic studies.

Thus it appears to the present author that infrared emission spectroscopy of carbon and other plasmas have interesting future.

\section{References}

1. Kroto HW, Heath JR, O'Brien SC, Curl RF, Smalley RE (1985) $\mathrm{C}_{60}$ : Buckminster fullerene. Nature 318: 162-163.

2. Kraetschmer W, Lamb LD, Fostiropoulos K, Huffman DR (1990) Solid C $:$ : A new form of carbon. Nature 347: 354-358.

3. Henning TH (1998) Carbon in the universe. Science 282: 2204-2210.

4. https://en.wikipedia.org/wiki/Allotropes_of_carbon

5. Groenewegen MAT (1998) Infrared emission of carbon stars. Astrophysics and Space Science Library 230: 73-86.

6. Yang CSC, Brown E, Hommerich U, Sudhir BT, Alan CS, et al. (2008) Midinfrared laser-induced breakdown spectroscopy emission from alkali metal halides. Applied Spectr. 62: 714-716.

7. Oyebola O, Hommerich U, Brown E, Yang C, Trivedi S, et al. (2011) Concentration dependent studies on the Laser-Induced Mid-infrared Emission from KCl-NaCl Tablets. Photonic Lett. Poland 3: 171-174.

8. Yang CSC, Brown E, Hommerich U, Jin F, Sudir BT, et al. (2012) Longwave, infrared laser-induced breakdown (LIBS) spectroscopy emissions from energetic materials. Applied Spectr. 66: 1397-1402.

9. Yang CS, Brown E, Kum-Barimah E, Hommerich UH, Jin F, et al. (2014) Midinfrared, long wave infrared $(4-12 \mu \mathrm{m})$ molecular emission signatures from pharmaceuticals using laser-induced breakdown spectroscopy (LIBS). Applied Spectr. 68: 226-231.

10. Nemes L, Brown E, Yang CS (2017) Mid-infrared emission spectroscopy of carbon plasma. Spectrochim. Acta Part A. 170: 145-149.

11. Frum Cl, Engleman R, Hedderich HG, Bernath PF (1991) The Infrared Emission spectrum of gas-phase $\mathrm{C}_{60}$ (Buckminster fullerene). Chem Phys Lett. 176: 504-508.

12. Nemes L, Ram RS, Bernath PF, Tinker FA (1994) Gas-phase infrared emission spectra of $\mathrm{C}_{60}$ and $\mathrm{C}_{70}:$ Temperature dependent studies. Chem Phys Lett. 218 295-303.

13. Chase $B$, Herron $N$, Holler $E$ (1992) Vibrational spectroscopy of fullerenes $\left(C_{60}\right.$ and $\mathrm{C}_{70}$ ) Temperature dependant studies. J Phys Chem. 96: 4262.

14. Dresselhaus MS, Dresselhaus G, Eklund PC (1995) Science of fullerenes and carbon nanotubes, their properties and applications. Academic Press. New York, USA.

15. Haufler RE, Conceicao J, Chibante LPF, Chai Y, Byrne NE, et al. (1990) Efficient production of $\mathrm{C}_{60}$ (buckminster fullerene) $\mathrm{C}_{60} \mathrm{H}_{36}$ and the solvated buckide ion. J Phys Chem. 94: 8634.

16. Cami J, Bernard-Salas J, Peeters E, Malek SE (2010) Detection of $C_{60}$ and $C_{70}$ in a young planetary nebula. Science 329: 1180-1182.

17. Garcia-Hernandez DA, Manchado A, Garcia-Lario P, Stanghellini L, Villaver $\mathrm{E}$, et al. (2010) Formation of fullerenes in $\mathrm{H}$-containing planetary nebulae. Astrophys J Lett. 724: 39-43.

18. Houck JR, Roellig TL, Van Cleve J, Forrest WJ, Herter T (2004) The infrared spectrograph (irs) on the spitzer space telescope. The Astrophysical Journal Supplement Series 154: 18

19. Yang CS, Jin F, Trivedi S, Brown E, Hommerich U, et al. (2016) Time-resolved long-wave infrared laser-induced breakdown spectroscopy of inorganic energetic materials by a rapid mercury-cadmium-telluride linear array detection system. Appl Opt. 55: 9166-9171.

20. Yang CSC, Brown E, Kumi-Barimah E, Hommerich U, Jin F, et al. (2015) Rapid long-wave infrared laser-induced breakdown spectroscopy measurements using a mercury-cadmium-telluride linear array detection system. Appl Opt. 54 9695-9702.

21. Kramida A, Ralchenko Y, Reader J (2014) NIST ASD Team. NIST Atomic Spectra Database (version 5.2)

22. Mackie CJ, Peeters E, Bauschlicher CW, Cami J (2015) Characterizing the infrared spectra of small neutral Fully Dehydrogenated Polycyclic Aromatic Hydrocarbons. Astrophys J 799: 1-11.

23. Kumar SK, Peeters FM (2013) Vibrational properties of nanographene Nanoscale Systems 2: 10-29.

24. Vandescuren M, Hermet P, Meunier V, Henrard L, Lambin PH (2008) Theoretical study of the vibrational edge modes in graphene nanoribbons. Phys Rev B 78: 195401.

25. Strelnikov D, Reusch R, Kraetschmer W (2005) Assignment of carbon containing molecules in cryogenic matrices by selective laser-induced oxidation. J Phys Chem 109: 7708-7713.

26. Chaffee CD (2017) LIBS continues to evolve. Optics and Photonics News pp: $42-49$.

27. James Webb Space Telescope. https://www.jwst.nasa.gov/ 